\title{
Hydrocephalus Following Bilateral Dumbbell- Shaped C2 Spinal Neurofibromas Resection and Postoperative Cervical Pseudomeningocele in a Patient with Neurofibromatosis Type 1: A Case Report
}

\author{
Nicola Montemurro ${ }^{1}$ Ardico Cocciaro ${ }^{1}$ Antonio Meola ${ }^{1}$ Ludovico Lutzemberger ${ }^{1}$ Riccardo Vannozzi ${ }^{1}$ \\ 1 Department of Neurosurgery, Azienda Ospedaliero Universitaria \\ Pisana, Pisa, Italy \\ Evid Based Spine Care J 2014;5:136-138. \\ Address for correspondence Nicola Montemurro, MD, Department of \\ Neurosurgery, Azienda Ospedaliero Universitaria Pisana, Via Paradisa, \\ 2, 56100 Pisa, Italy (e-mail: montemurronic@hotmail.com).
}

\author{
Abstract \\ Keywords \\ - spinal cord tumor \\ - cervical spine \\ - dumbbell-shaped \\ neurofibroma \\ - hydrocephalus \\ - postoperative cervical \\ pseudomeningocele \\ - neurofibromatosis \\ type 1
}

Study Design Case report.

Objective To present a rare case of hydrocephalus following bilateral dumbbellshaped C2 spinal neurofibromas resection and postoperative cervical pseudomeningocele in a patient with neurofibromatosis type 1 (NF1).

Methods The patient's clinical course is retrospectively reviewed. A 37-year-old man affected by NF1 referred to our department for progressive weakness of both lower extremities and gait disturbance. Radiological imaging showed bilateral dumbbellshaped C2 spinal neurofibromas. After its resection, at the 1-month follow-up evaluation, the patient reported headache and nausea. A CT brain scan showed a postoperative cervical pseudomeningocele and an increase in the ventricular sizes, resulting in hydrocephalus.

Results A ventriculoperitoneal shunting was performed using a programmable valve opening pressure set to $120 \mathrm{mmH}_{2} 0$. After surgery, the patient's neurological status markedly improved.

Conclusion Hydrocephalus must be considered a possible complication of cervical spine tumor resection.

\section{Introduction}

Although reports of the coexistence of hydrocephalus and neurofibromatosis type 1 (NF1) have already been published, and cervical spine neurofibromas in patients with NF1 are common, a bilateral dumbbell-shaped C2 spinal roots location and the onset of hydrocephalus following its resection are not.

\section{Case Report}

We present a 37-year-old man affected by NF1, referred to our department for progressive weakness of both lower extremities

received

February 23, 2014

accepted

July 2, 2014

and gait disturbance. A physical examination revealed a 4 to 5/5 muscle strength in lower limbs and hypesthesia below the C3 level. Magnetic resonance imaging (MRI) of the cervical spine disclosed a bilateral dumbbell-shaped enhancing lesion located around the $\mathrm{C} 1$ and $\mathrm{C} 2$ segments, compressing the spinal cord posteriorly (-Fig. 1). The patient underwent C1-C2 laminectomy through a posterior approach, and both sides of the tumors were resected intralesionally (-Fig. 2). Pathological examination revealed neurofibromas. After surgery, we found improved motor function. However, at the 1-month follow-up evaluation, the patient complained of headaches and nausea. A CT scan of the brain showed a postoperative cervical

(c) 2014 Georg Thieme Verlag KG Stuttgart · New York
DOI http://dx.doi.org/ 10.1055/s-0034-1387805. ISSN 1663-7976. 


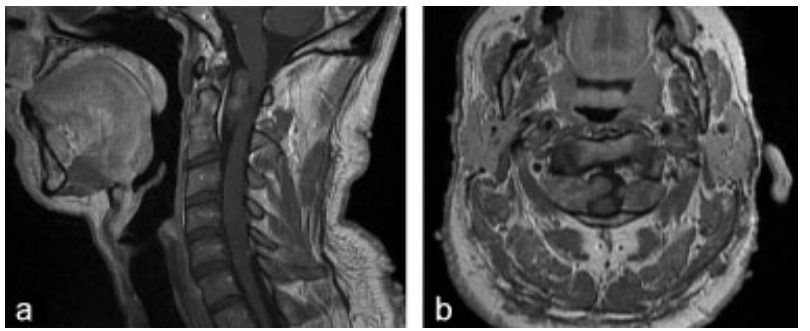

Fig. 1 Preoperative sagittal (a) and axial (b) T1-weighted gadoliniumenhanced MRI scan showing a bilateral C1-C2 dumbbell-shaped enhancing lesion. MRI, magnetic resonance imaging.

pseudomeningocele and an increase in the ventricular sizes, resulting in hydrocephalus (-Fig. 3a, b). Ventriculoperitoneal shunting was performed using a programmable valve opening pressure set to $120 \mathrm{mmH}_{2} 0$. After surgery, the patient's neurological status markedly improved. CT cervical and brain scans

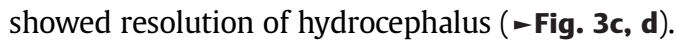

\section{Discussion}

NF1, also known as von Recklinghausen disease, is a genetic autosomal dominant disorder caused by the mutation of a gene on chromosome 17 that is responsible for the control of cell division. According to $\mathrm{NIH}$ Consensus Development Conference Criteria, clinical manifestations of NF1 are neurofibromas, multiple café-au-lait spots, axillary freckling, Lisch nodules on the iris, and intracerebral hamartomas. ${ }^{1}$ It is estimated that in $\sim 2 \%$ of patients with NF1, symptomatic spinal nerve root tumors develop. $^{2}$ In patients with NF1, cervical spine neurofibromas are common and even bilateral dumbbell-shaped cervical spine roots locations are not so rare., ${ }^{3,4}$ The coexistence of hydrocephalus and NF1 has already been published, even if the main causes for hydrocephalus at initial presentation associated with NF1
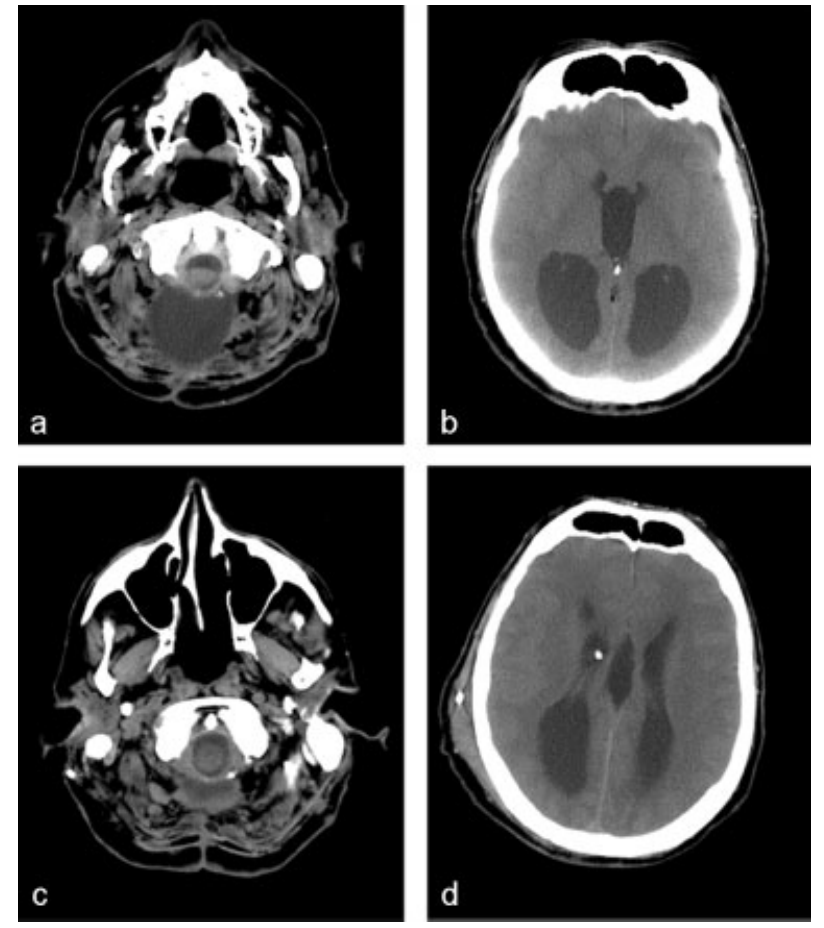

Fig. 3 Postoperative CT brain scan showing a postoperative cervical pseudomeningocele with ventricular size enlarged $(a, b)$ and subsequent resolution of hydrocephalus after a ventriculoperitoneal shunting (c, d). CT, computed tomography.

were reported as posterior fossa tumors, aqueduct stenosis due to periaqueductal/tectal hamartomas, and rarely brain stem glioma or tectal glioma. ${ }^{5-8}$ Hydrocephalus at initial presentation due to intraspinal tumors is well-known, even if it is a rare condition, since $\sim 1 \%$ of patients with spinal cord tumors have various degrees of hydrocephalus. ${ }^{9}$ The pathogenesis of hydrocephalus reported as a result of spinal tumor pathology is caused
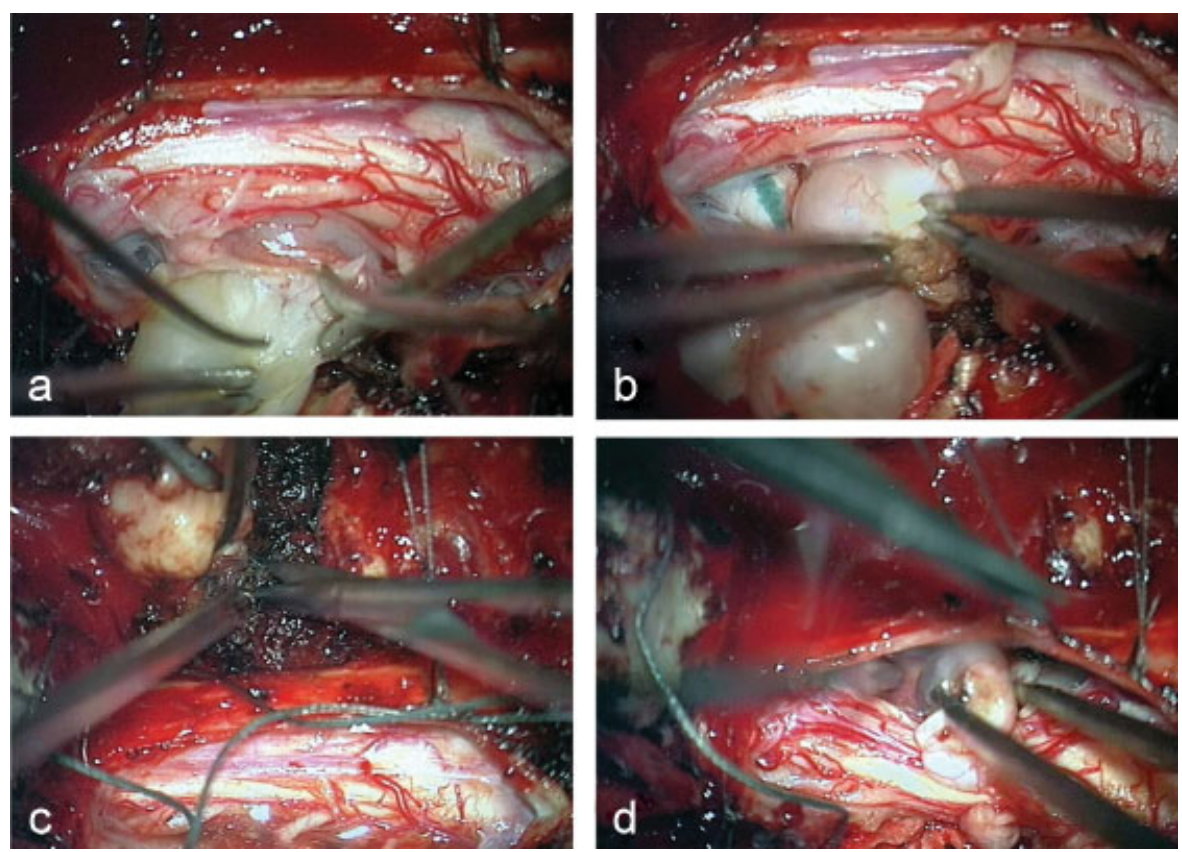

Fig. 2 Operative view showing right neurofibroma removal (a, b) and left tumor excision (c, d) resecting C2 nerve roots, after C1-C2 spinolaminectomy through a posterior approach. 
by a disruption of the cerebrospinal fluid (CSF) flow, impaired absorption due to elevated CSF protein content, tumor infiltration into basal cisterns, or compression of the spinal venous plexus. ${ }^{10,11}$ In our case, there was no residual cervical spine tumor, and the hydrocephalus, which was not present at initial presentation, could be a consequence of CSF flow obstruction due to cervical postoperative pseudomeningocele. In our opinion, a tear in the dura and the following accumulation of CSF in the epidural space may be found in a ball valve mechanism allowing egress of CSF and resulting in a tense pseudomeningocele, which causes obstruction of free CSF and hydrocephalus.

In summary, we report an unusual case of hydrocephalus following bilateral dumbbell-shaped C2 spinal neurofibromas resection and postoperative cervical pseudomeningocele in patients with NF1. Surgical treatment of cervical dumbbellshaped neurofibromas is highly successful due to the benign nature of the tumors. Our case report focuses on a possible complication of cervical spine tumor resections. Whenever a postoperative pseudomeningocele occurs, the possibility of concurrent hydrocephalus may have to be considered; neurological examination can help in obtaining the correct diagnosis and deciding the best surgical strategy. After surgical treatment, a long-term clinical and radiological follow-up is mandatory.

\author{
Disclosures \\ Nicola Montemurro, none \\ Ardico Cocciaro, none \\ Antonio Meola, none \\ Ludovico Lutzemberger, none \\ Riccardo Vannozzi, none
}

\section{References}

1 Conference NIHCD; National Institutes of Health Consensus Development Conference. Neurofibromatosis. Conference statement. Arch Neurol 1988;45(5):575-578

2 Bartolomei JC, Crockard HA. Bilateral posterolateral approach to mirror-image C-2 neurofibromas. Report of four cases. J Neurosurg 2001;94(2, Suppl)292-298

3 Taleb FS, Guha A, Arnold PM, Fehlings MG, Massicotte EM. Surgical management of cervical spine manifestations of neurofibromatosis Type 1: long-term clinical and radiological follow-up in 22 cases. J Neurosurg Spine 2011;14(3):356-366

4 Junming M, Cheng Y, Dong C, et al. Giant cell tumor of the cervical spine: a series of 22 cases and outcomes. Spine (Phila Pa 1976) 2008;33(3):280-288

5 Dinçer A, Yener U, Özek MM. Hydrocephalus in patients with neurofibromatosis type 1: MR imaging findings and the outcome of endoscopic third ventriculostomy. AJNR Am J Neuroradiol 2011; 32(4):643-646

6 Hosoda K, Kanazawa Y, Tanaka J, Tamaki N, Matsumoto S. Neurofibromatosis presenting with aqueductal stenosis due to a tumor of the aqueduct: case report. Neurosurgery 1986;19(6): 1035-1037

7 Pollack IF, Shultz B, Mulvihill JJ. The management of brainstem gliomas in patients with neurofibromatosis 1. Neurology 1996; 46(6):1652-1660

8 Créange A, Zeller J, Rostaing-Rigattieri S, et al. Neurological complications of neurofibromatosis type 1 in adulthood. Brain 1999;122(Pt 3):473-481

9 Mirone G, Cinalli G, Spennato P, Ruggiero C, Aliberti F. Hydrocephalus and spinal cord tumors: a review. Childs Nerv Syst 2011; 27(10):1741-1749

10 Rifkinson-Mann S, Wisoff JH, Epstein F. The association of hydrocephalus with intramedullary spinal cord tumors: a series of 25 patients. Neurosurgery 1990;27(5):749-754, discussion 754

11 Cohen AR, Wisoff JH, Allen JC, Epstein F. Malignant astrocytomas of the spinal cord. J Neurosurg 1989;70(1):50-54

\section{Editorial Perspective}

The submission by Montemurro et al deserved publication in EBSJ due to the rarity and severity of the underlying pathology in this location and for the learning points it can provide for our readers.

As identified by Dr. Dailey in the commentary, there are questions to be answered prior to drawing larger conclusions about such Neurofibroma resections. To date, there is little or no guidance provided by the literature in terms of the need for preoperative imaging and restitution of CSF flow around a larger, neural element tumor resection, such as done here. This raises the question of obtaining preoperative baseline cranial imaging in such neural element tumor resections and the need for early postoperative imaging. Based on Dr Dailey's insights, it appears reasonable to suggest routine preoperative cranial imaging for patients with spinal cord tumors in light of a $1 \%$ incidence of concurrent intracranial pathology. Further, the nature of the dural reconstruction post-resection and reestablishing contained CSF flow are intraoperative considerations that are surgeon specific and not really amenable to literature guidance. EBSJ invites its readership to comment on the need for preemptive shunting and dural reconstruction in tumor resections like the one presented here. 